\title{
ALTERAÇÕES MICROBIOLÓGICAS DO SOLO APÓS TRÊS ANOS DE SISTEMA INTEGRAÇÃO LAVOURA -PECUÁRIA SOB DIFERENTES ADUBAÇÕES E ESCARIFICAÇÃO DO SOLO
}

\author{
Daniele Perreti Bettio, Fábio Fernando de Araújo \\ Universidade do Oeste Paulista - UNOESTE, Mestrado em Agronomia - Produção Vegetal, Presidente \\ Prudente, SP. E-mail: dpbettio@gmail.com
}

\begin{abstract}
RESUMO
A partir da implantação do sistema de integração lavoura -pecuária, desde 2013, em uma área na região do Oeste Paulista, o presente trabalho teve como objetivo avaliar mudanças microbiológicas no solo do local. Foram coletadas amostras de solo da área experimental a campo implantada em delineamento experimental inteiramente casualizado em sistema fatorial duplo, (2x5) com cinco adubações. Os tratamentos consistiram em escarificação do solo e diferentes adubações: $\mathrm{TO}=$ Controle; $\mathrm{T} 1$ = Calcário; $\mathrm{T} 2=$ Calcário + gesso agrícola; $\mathrm{T} 3=$ Calcário + gesso agrícola + NPK; e T4= Calcário + gesso agrícola + NPK + micronutrientes. Realizou-se análises de biomassa e respiração do solo e os resultados foram submetidos ao Teste de Tukey. A biomassa microbiana do solo, a respiração basal e o qCO2 foram influenciados pela escarificação e adubação mineral do solo, sendo que na área escarificada a perda de carbono foi mais elevada, sugerindo um desequilíbrio microbiano no sistema.
\end{abstract}

Palavras - chave: Indicadores biológicos. Carbono orgânico. Biomassa microbiana.

\section{CHANGES OF SOIL MICROBIOLOGICAL AFTER INTEGRATION SYSTEM THREE YEARS CROP - LIVESTOCK UNDER DIFFERENT FERTILIZATION AND SOIL SCARIFICATION}

\begin{abstract}
From the implementation of crop-livestock system since 2013 in an area in the West Paulista region, the present study was to evaluate microbiological changes in soil site. Soil samples were collected from the experimental area located field in a completely randomized design in double factorial system $(2 \times 5)$ with five fertilization. The treatments consisted of soil scarification and different fertilizations: $\mathrm{TO}=$ control; $\mathrm{T} 1$ = Limestone; $\mathrm{T} 2$ = lime + gypsum; $\mathrm{T} 3$ = lime + gypsum + NPK; and T4 = lime + gypsum + NPK + micronutrients. We conducted analysis of biomass and soil respiration and the results were submitted to Tukey test. The soil microbial biomass, basal respiration and qCO2 were influenced by scarification and mineral soil fertilization, and in the area scarified carbon loss was higher, suggesting a microbial imbalance in the system.
\end{abstract}

Keywords: Biological indicators. Organic carbon. Microbial biomass. 


\section{INTRODUÇÃO}

Com a alta demanda alimentícia e a baixa capacidade que alguns solos têm em produzir alimentos devido às suas características tais como os solos arenosos, sistemas de cultivos eficientes vêm sendo adotados, entre ele a integração lavoura- pecuária (ILP). Assim, ILP se conceitua como um sistema de produção que agrega vários fatores, tais como, biológicos, econômicos e sociais que se interagem, mitigando os impactos ambientais (JUNIOR et al., 2009).

Portanto o uso do monitoramento das atividades biológicas do solo e suas modificações se tornam um importante aliado para diagnosticar eventuais mudanças ocasionadas pelo uso de diferentes sistemas agrícolas (DA SILVA OLIVEIRA et al., 2015). Existem indicadores de qualidade do solo, isto é, atributos que podem medir e refletir no status ambiental, dentre eles temos indicadores químicos e físicos e não menos importantes os biológicos do solo, pois os microrganismos são peças chaves por serem sensíveis às rápidas alterações no solo (DE ARAÚJO; MONTEIRO, 2007), permitindo assim que precauções antecipadas sejam tomadas, contribuindo para o aumento da sustentabilidade do sistema de produção (TÓTOLA; CHAER 2002).

Entre os bioindicadores de qualidade do solo temos a biomassa microbiana, esta consiste como a parte viva da matéria orgânica do solo, compreendendo de 1 a $5 \%$ do carbono orgânico total do solo (JENKINSON; LADD, 1981). A biomassa microbiana é um dos principais componentes que controlam variadas funções do solo, representando a decomposição e o acúmulo de matéria orgânica, além de ser uma reserva rica de nutrientes, não só estocando, mas também ciclando os nutrientes no sistema (GREGORICH et al., 1994). Tem-se também a taxa respiratória dos microrganismos, que mede a degradação dos compostos orgânicos a CO2 (SILVA et al., 2013), e pode ser influenciada por diversos fatores como umidade, textura, estrutura do solo e quantidade de matéria orgânica, já que estes refletirão sobre a atividade metabólica e fisiológica das células microbianas interferindo na respiração basal dos mesmos (SILVA et al, 2010).

Por fim, apenas um indicador não é capaz de relacionar todas as mudanças no ambiente, por isso temos a correlação entre a respiração e a biomassa microbiana representada pelo quociente metabólico (qCO2), ele expressa a quantidade de $\mathrm{CO} 2$ liberado a partir da biomassa microbiana em função de um tempo determinado, isto é, a taxa de respiração específica da biomassa microbiana (ALVES et al.,2011).

O objetivo do presente trabalho foi avaliar as mudanças biológicas do solo a partir da biomassa microbiana, da respiração basal e do qCO2, em um sistema de integração lavoura pecuária implantado na região do Oeste Paulista no terceiro ano de cultivo, com sucessão de pastagem e soja, sob diferentes adubações e escarificação do solo.

\section{METODOLOGIA}

Em 2013 foi implantado em uma área de 5,3 ha na fazenda experimental da UNOESTE, em Presidente Bernardes - SP um experimento com sistema de integração lavoura - pecuária (ILP), sendo o solo desta área caracterizado como argissolo vermelho distroférrico, no qual apresenta um maior acúmulo de argila em profundidade (horizonte b) maior do que na camada superficial do solo (EMBRAPA 2006).

A área experimental foi dividida em duas sub-áreas e em 2013 foi realizada a escarificação apenas de uma delas, essas sub-áreas foram divididas em 5 parcelas, onde cada uma delas teve um manejo mineral (adubação), sendo portanto 5 adubações diferentes, definidas como: T0 = Controle (apenas adubação de manutenção); T1 = Calcário; T2= Calcário + gesso agrícola; T3= Calcário + gesso agrícola + NPK; e T4= Calcário + gesso agrícola + NPK + micronutrientes; ressaltase que essas adubações foram realizadas antes do semeio e ressemeio anual da pastagem (Urochloa brizantha) na área, seguindo recomendações técnicas e em ambos os tratamentos também se realizou a adubação de manutenção da cultura de grãos, que no caso era soja.

No dia 20 de outubro de 2015 foi realizada a coleta do solo de cada tratamento, com auxílio de uma cavadeira, cada faixa foi dividida supostamente em 6 sub-faixas e dentro de cada 
uma delas foram coletadas $5 \mathrm{sub}$ - amostras, em uma profundidade de aproximadamente $15 \mathrm{~cm}$, que quando juntas formaram uma repetição. Portanto o delineamento experimental utilizado foi inteiramente casualizado, com 2 manejos físicos (escarificação) e 5 adubações ( $2 \times 5$ ), formando 10 tratamento com 6 repetições.

Para análise do carbono da biomassa microbiana ( $\mathrm{Cmic}$ ) se utilizou do método da fumigação-extração (VANCE et al., 1987), sendo o fator de conversão da fórmula 0,38, segundo Feigl et al., 1995, sendo o Cmic expresso em mg C kg- ${ }^{1}$ de solo. Já a respiração do solo foi avaliada segundo Alef e Nanipieri (1995) com algumas modificações, empregando-se o método condutimétrico (RODELLA; SABOYA, 1999), com leitura em 24 e 48 horas. A quantidade de CO2 produzida pela respiração foi calculada utilizando-se a fórmula definida por Rodella e Saboya (1999) e os resultados foram expressos em mg de C-CO2 kg-1 h-1. E por fim o quociente metabólico (qCO2) foi definido a partir da relação entre a taxa respiratória e o carbono da biomassa microbiana, sua unidade foi expressa em mg C-CO2 $\mathrm{kg}^{-1} \mathrm{CO} 2 \mathrm{dia}-{ }^{-1}$ (ANDERSON; DOMSCH, 1993).

Ambos os resultados foram submetidos à análise de variância pelo Teste de Tukey, em comparação de médias, ao nível de $5 \%$ de probabilidade utilizando o programa ASSISTAT versão 7.7 beta.

\section{RESULTADOS}

Atributos biológicos do solo devem ser estudados e levados em consideração quando se pretende entender as mudanças nos ambientes de produção, de tal forma, observando a tabela 01, podemos constatar que a não escarificação da área agrícola, de modo geral, aumentou a quantidade de biomassa microbiana no solo, e as adubações com calagem, calagem + gesso agrícola e ambas às adubações citadas anteriormente com NPK foram às responsáveis por maiores valores de carbono microbiano presente no solo.

Tabela 01. Biomassa microbiana (mg $\mathrm{C} \mathrm{Kg}^{-1}$ solo) após três anos de integração lavoura - pecuária submetida a diferentes adubações minerais e manejo físico do solo (escarificação) em amostras de solo coletadas após cultivo de pastagem.

\begin{tabular}{|c|c|c|c|}
\hline \multirow{2}{*}{ Adubações } & \multicolumn{2}{|c|}{ Escarificação } & \multirow[b]{2}{*}{ Médias } \\
\hline & Sem & Com & \\
\hline & \multicolumn{2}{|c|}{ mg C Kg- ${ }^{1}$ solo } & \\
\hline Controle ${ }^{1}$ & $67,60 \mathrm{cA}$ & $62,04 \mathrm{cA}$ & $64,82 \mathrm{~b}$ \\
\hline Calagem $^{2}$ & 147,13 aA & $88,25 \mathrm{bB}$ & 117,69 a \\
\hline Calagem + Gesso agrícola ${ }^{2}$ & $128,04 \mathrm{abA}$ & $113,41 \mathrm{aA}$ & $120,72 \mathrm{a}$ \\
\hline Cal. + Ges. + NPK ${ }^{2}$ & $106,66 \mathrm{bA}$ & $120,50 \mathrm{aA}$ & $113,58 \mathrm{a}$ \\
\hline Cal.+ Ges. +NPK + Micros ${ }^{2}$ & $35,82 \mathrm{dA}$ & $43,28 \mathrm{cA}$ & $39,55 \mathrm{c}$ \\
\hline
\end{tabular}

Médias

$97,05 \mathrm{~A}$

85,49 B

${ }^{1}$ Apenas com adubação de plantio da cultura anual; ${ }^{2}$ Todos receberam adubação de plantio da cultura anual mais as respectivas adubações minerais descritas; Letras maiúsculas são referentes aos valores das colunas e letras minúsculas aos valores das linhas. Letras iguais não se diferem ao Teste de Tukey ao nível de $5 \%$ de probabilidade.

A taxa respiratória foi maior nos tratamentos com correções e/ou adubações do solo sem escarificação (Tabela 02), pois a liberação de $\mathrm{CO} 2$ no tratamento controle, que teve o solo revolvido foi elevada ultrapassando as demais, exceto para a adubação de calagem + gesso agrícola + NPK. 
Tabela 02 - Quantificação da liberação de $\mathrm{CO}_{2}$ do solo através da análise de respiração microbiana em um ambiente de integração lavoura - pecuária após o terceiro ano de implantação do sistema.

\begin{tabular}{|c|c|c|c|}
\hline \multirow{2}{*}{ Adubações } & \multicolumn{2}{|c|}{ Escarificação } & \multirow[b]{2}{*}{ Médias } \\
\hline & Sem & Com & \\
\hline & \multicolumn{2}{|c|}{$\mathrm{mg}$ de $\mathrm{C}-\mathrm{CO}_{2} \mathrm{~kg}^{-1}$ solo $\mathrm{h}^{-1}$} & \\
\hline Controle $^{1}$ & $2,87 \mathrm{bB}$ & 6,68 aA & $4,78 a b$ \\
\hline Calagem $^{2}$ & 4,36 aA & $3,10 \mathrm{bB}$ & $3,77 \mathrm{~b}$ \\
\hline Calagem + Gesso agrícola² & 5,09 aA & $3,08 \mathrm{bB}$ & $4,08 a b$ \\
\hline Cal. + Ges. + NPK ${ }^{2}$ & 4,26 abA & 5,40 aA & $4,83 a$ \\
\hline Cal.+ Ges. +NPK + Micros 2 & $3,74 \mathrm{abA}$ & $3,76 \mathrm{bA}$ & $3,75 \mathrm{c}$ \\
\hline
\end{tabular}

Médias 4,06 A 4,42 A

\footnotetext{
${ }^{1}$ Apenas com adubação de plantio da cultura anual; ${ }^{2}$ Todas receberam adubação de plantio da cultura anual mais as respectivas adubações minerais descritas; Letras maiúsculas são referentes aos valores das colunas e letras minúsculas aos valores das linhas. Letras iguais não se diferem ao Teste de Tukey ao nível de $5 \%$ de probabilidade.
}

O quociente metabólico (qCO2), foi maior no tratamento com todas as fontes de adubos (calagem + gesso agrícola + NPK + micros) na área não escarificada e foi inferior apenas para o controle na área escarificada (Tabela 03), sendo que os tratamentos que tiveram alta quantidade de biomassa microbiana apresentaram menores perdas de carbono pela respiração, já que o qCO2 destes foram inferiores. Ressalta-se por sua vez que o tratamento controle na área escarificada, no tempo em que foi estudado, foi um ambiente desfavorável aos microrganismos, já que não elevou a biomassa e refletiu em maiores perdas de carbono.

Tabela 03 - Quociente metabólico $\left(\mathrm{qCO}_{2}\right)$, a relação entre taxa respiratória e biomassa microbiana de um solo arenoso após terceiro de sistema de integração lavoura - pecuária submetido a diferentes adubações e escarificação.

\begin{tabular}{|c|c|c|c|}
\hline \multirow{2}{*}{ Adubações } & \multicolumn{2}{|c|}{ Escarificação } & \multirow[b]{2}{*}{ Médias } \\
\hline & Sem & Com & \\
\hline & \multicolumn{2}{|c|}{$\mathrm{mg}$ de $\mathrm{C}-\mathrm{CO}_{2} \mathrm{~kg}^{-1} \mathrm{CO}_{2} \mathrm{dia}^{-1}$} & \\
\hline Controle $^{1}$ & $0,068 \mathrm{bB}$ & $0,215 \mathrm{aA}$ & $0,141 b$ \\
\hline Calagem $^{2}$ & $0,056 \mathrm{bA}$ & $0,061 \mathrm{dA}$ & $0,058 d$ \\
\hline Calagem + Gesso agrícola ${ }^{2}$ & $0,063 \mathrm{bA}$ & 0,032 eB & $0,048 d$ \\
\hline Cal. + Ges. + NPK ${ }^{2}$ & $0,074 \mathrm{bB}$ & $0,107 \mathrm{cA}$ & $0,091 \mathrm{c}$ \\
\hline Cal.+ Ges. +NPK + Micros ${ }^{2}$ & 0,245 aA & $0,149 \mathrm{bB}$ & $0,197 \mathrm{a}$ \\
\hline Médias & $0,101 \mathrm{~B}$ & $0,113 \mathrm{~A}$ & \\
\hline
\end{tabular}




\section{DISCUSSÃO}

Alterações ocasionadas pelos sistemas de produção no solo podem alterar diretamente a estrutura e a atividade biológica e consequentemente sua fertilidade (BROOKES, 1995). Entretanto, condições distintas em que o solo se encontra sejam sob vegetação e até mesmo manejos físicos, tais como, perturbações do solo decorrente de práticas agrícolas, tendem a influenciar nas taxas de biomassa microbiana, pois podem diminuir a quantidade de carbono da biomassa do solo ou aumentar (D' ANDRÉA et al., 2002). Estudo como o de Carneiro et al., (2008), demonstraram que em sistema de integração lavoura - pecuária houve aumentos de até $50 \%$ no valor de Cmic, em relação as áreas nativas de cerrado, sugerindo que o sistema radicular da gramínea favoreça a microbiota.

Assim, no tocante em que se refere as práticas agrícolas, Doran (1980) relatou que onde existe menor revolvimento do solo, isto é, plantio direto, há uma melhoria nas condições da rizosfera influenciando em maiores valores de populações microbianas do que em sistemas convencionais, confirmando de fato os valores obtidos no solo escarificado, ao ter influenciado de maneira negativa na microbiologia do solo no presente trabalho. Ressalta-se que onde há baixos valores de biomassa microbiana e altos valores de respiração, o caso do solo na área escarificada, pode significar, em curto prazo, um fator limitante já que aumenta a liberação de nutrientes para as plantas, porém em longo prazo irá ocasionar uma perda de carbono do solo elevada para a atmosfera (PARKIN et al., 1996).

A respiração basal representa a oxidação da matéria orgânica presente no sistema pelos organismos aeróbicos, enquanto a relação respiração e biomassa que nos referimos como qCO2 expressa a quantidade de carbono que é oxidado por um certo tempo através da biomassa microbiana (NEVES et al., 2009). No caso de sistemas com revolvimento do solo, a perturbação contribui para um estresse na população dos microrganismos e uma vez que a quantidade de carbono é menor nessas áreas, em relação ao sistema sem revolvimento, a biomassa microbiana tende a consumir mais carbono orgânico do solo, causando sua redução (NEVES etal.,2009), tal afirmação tende a explicar o fato de que no solo escarificado o valor do Cmic foi menor, porém a respiração basal se elevou.

Entende-se ainda que os valores de qCO2 encontrados no trabalho podem ser explicados pelo fato de que valores elevados de qCO2 demonstram que está ocorrendo uma alta taxa de consumo de carbono oxidável para a manutenção da população microbiana (ANDERSON; DOMSCH, 1993). Por outro lado, baixo qCO2 indica um ambiente mais estável e mais próximo de seu equilíbrio (TOTÓLA; CHAER, 2002).

Por fim houve alterações ocasionada devido a escarificação do solo e também por influência nutricional do solo devido as diferentes adubações, sendo que então o aspecto nutricional se mostrou pertinente ao proporcionar alterações microbiológicos, assim como sugerem os autores Schneider et al., (2011), que concluíram que a calagem do solo foi benéfica aos atributos de colonização micorrizica de fungos de solo, aumentando a microbiota, especialmente em sistema de plantio direto.

\section{CONCLUSÕES}

A biomassa microbiana do solo, a respiração basal e o qCO2 foram influenciados pela escarificação e adubação mineral do solo, sendo que na área escarificada ocorreu uma perda de carbono da matéria orgânica mais elevada, sugerindo um desequilíbrio microbiano no sistema.

\section{REFERÊNCIAS}

ANDERSON, J. P. E.; DOMSCH, K. H. The metabolic quotient of $\mathrm{CO} 2$ (q $\mathrm{CO} 2$ ) as a specific activity paramenter to assess the effects of environmental condition, such as $\mathrm{pH}$, on the microbial of forest soil. Soil Biology and Biochemistry, v. 25, n. 3, p. 393-395, 1993. https://doi.org/10.1016/0038-0717(93)90140-7 
ALEF, K.; NANNIPIERI, P. Methods in applied soil microbiology and biochemistry. London: Academic Press, p.576, 1995.

BROOKES, P. C. The use of microbial parameters in monitoring soil pollution by heavy metals. Biology and Fertility of soils, v. 19, n. 4, p. 269-279, 1995. https://doi.org/10.1007/BF00336094

CARNEIRO, M.A.C.; ASSIS, P.C.R.; MELO, L.B. de C.; PEREIRA, H.S.; PAULINO, H.B.; SILVEIRA NETO, A.N. da. Atributos bioquímicos em dois solos de cerrado sob diferentes sistemas de manejo e uso. Pesquisa Agropecuária Tropical, v.38, p.276-283, 2008.

D'ANDRÉA, A.F.; SILVA, M.L.N.; CURI, N.; SIQUEIRA, J.O.; CARNEIRO, M.A.C. Atributos biológicos indicadores da qualidade do solo em sistemas de manejo na região do cerrado no sul do estado de Goiás. Revista Brasileira de Ciência do Solo, v. 26, n. 4, p. 913-924, 2002. https://doi.org/10.1590/S0100-06832002000400008

DA SILVA OLIVEIRA, B., DE CARVALHO, M. A. C., LANGE, A., WRUCK, F. J., DALLACORT, R. Atributos biológicos do solo em sistema de integração lavoura-pecuária-floresta, na região amazônica. Revista Engenharia na Agricultura, v. 23, n. 5, p. 448, 2015. https://doi.org/10.13083/1414-3984/reveng.v23n5p448-456

DE ARAÚJO, FERREIRA A.S.; MONTEIRO,R. T. S.. Indicadores biológicos de qualidade do solo. Bioscience Journal, v. 23, n. 3, 2007.

DORAN, John W. Soil microbial and biochemical changes associated with reduced tillage. Soil Science Society of America Journal, v. 44, n. 4, p. 765-771, 1980. https://doi.org/10.2136/sssaj1980.03615995004400040022x

EMBRAPA SOLOS. Sistema Brasileiro de Classificação de Solos. 2. ed. Rio de Janeiro, 306 p. il. 2006.

FEIGL, B. J. et al. Soil microbial biomass in Amazonian soils: evaluation of methods and estimates of pool sizes. Soil Biology and Biochemistry, v. 27, n. 11, p. 1467-1472, 1995. https://doi.org/10.1016/0038-0717(95)00063-K

GREGORICH, E. G.; CARTER, M. R.; ANGERS, D. A.; MONREALL, C. M.; ELLERT, B. H. Towards a minimum data set to assess soil organic-matter quality in agricultural soils. Canadian Journal of Soil Science, Montreal, v. 74, p. 367-385, 1994. https://doi.org/10.4141/cjss94-051

JENKINSON, D. S.; LADD, J. N. Microbial biomass in soil: measurement and turnover. In: PAUL, E. A.; LADD, J. N. (Org.) Soil biochemistry. New York: Marcel Dekker. p. 415-471, 1981.

JUNIOR, A. B., MORAES, A., VEIGA, M., PELISSARI, A., \& DIECKOW, J. Integração lavoura-pecuária: intensificação de uso de áreas agrícolas. Ciência Rural, v.39, n.6, p.1925-1933, 2009. https://doi.org/10.1590/S0103-84782009005000107

NEVES, C. M. N., SILVA, M. L. N., CURI, N., MACEDO, R. L. G., MOREIRA, F. D. S., \& D'ANDRÉA, A. F. "Indicadores biológicos da qualidade do solo em sistema agrossilvipastoril no noroeste do Estado de Minas Gerais." Ciência e Agrotecnologia, v. 33 n.1, p.105-112, 2009. https://doi.org/10.1590/S1413-70542009000100015 
PARKIN, T.B.; DORAN, J.W.; FRANCOP-VIZCAÍNO, E. Field and laboratory tests of soil respiration. In: DORAN, J.W.; JONES, A. (Ed.). Methods for assessing soil quality. Madison: Soil Science Society of America, p.231-245. 1996.

RODELLA, A. A.; SABOYA, L. V. Calibration for conductimetric determination of carbon dioxide. Soil Biology and Biochemistry, v. 31, n. 14, p. 2059- 2060, 1999. https://doi.org/10.1016/S0038$\underline{0717(99) 00046-2}$

SCHNEIDER, J., KLAUBERG FILHO, O., FONTOURA, S. M., \& ALVES, M. V. Influência de diferentes sistemas de manejo e calagem em experimento de longa duração sobre fungos micorrízicos arbusculares. Ciência e Agrotecnologia, v. 35, n. 4, p. 701-709, 2011. https://doi.org/10.1590/S1413-70542011000400008

SILVA, J. C. D.; WENDLING, B.; CAMARGO, R. D.; MENDONÇA, L. B. P.; FREITAS, M. D. C. M. D. Analise comparativa entre os sistemas de preparo do solo: Aspectos técnicos e econômicos. Enciclopédia Biosfera, v. 7, n. 12, p. 1- 11, 2011.

SILVA, J. M.; ALBURQUERQUE, L. S. D.; SANTOS, T. M. C. D.; OlIVEIRA, J. U. L. D.; GUEDES, E. L. F. Mineralização de vermicompostos estimada pela respiração microbiana. Revista Verde, v. 8, n. 4, p. 132-135, 2013.

TÓTOLA, M.R.; CHAER, G.M. Microrganismos e processos microbiológicos como indicadores da qualidade do solo. In: ALVAREZ VENEGAS, V.H.; SCHAEFER, C.E.G.R.; BARROS, N.F. de; MELLO, J.W.V. de; COSTA, L.M. da. (Ed.). Tópicos em ciência do solo. Viçosa: Sociedade Brasileira de Ciência do Solo, p.195-276. 2002.

VANCE, E. D.; BROOKES, P. C.; JENKINSON, D. S. An extraction method for measuring soil microbial biomass C. Soil biology and Biochemistry, v. 19, n. 6, p. 703-707, 1987. https://doi.org/10.1016/0038-0717(87)90052-6 